\title{
New Radiation Formulae of Relativistic Electrons in Curved Magnetic Field Lines
}

\author{
Ya.M. Sobolev \\ Institute of Radio Astronomy, National Academy of Sciences of \\ Ukraine, 4 Chervonopraporna St., 310002, Kharkov,Ukraine
}

\begin{abstract}
Radiation mechanism for relativistc charged particles spiraling along curved magnetic field is considered. Emission from many electron revolutions around force line is taken into account.
\end{abstract}

Synchrotron radiation mechanism is among of the most important in radioastronomy. Curvature radiation, which has the same nature as synchrotron radiation, has been suggested as a pulsar radiation mechanism (Radhakrishnan 1969) and it is widely applied for explanation of observational data (Radhakrishnan \& Rankin 1990). In this case the formulae of synchrotron theory are used. But synchrotron radiation formulae, which are suitable only for the particles moving in uniform and straight magnetic fields, may be inadequate when particles move in a curved magnetic field. In particular in the interpretation of polarization data in extragalactic radio jets, or when one calculates the power radiated by relativistic particles in pulsar magnetosphere. Attempts (Cheng \& Zhang 1996; Sobolev 1999) have been made to describe the radiation of relativistic charged particles moving in curved magnetic field.

General radiation formulae for relativistic charged particles moving in curved magnetic field are obtained in the work. Parametric region, in which this radiation mechanism is applicable, is found for pulsar magnetosphere.

There is a charged particle (with Lorentz factor $\gamma \gg 1$ ) that exhibits a circular motion around the guiding center, which is moving with a constant speed along the circular magnetic field line. Synchrotron-curvature radiation (Cheng \& Zhang 1996) takes place as long as the radius of the curvature of the trajectory is comparable with the curvature radius $(R)$ of the magnetic field. We are considering here the case when the curvature radius of the trajectory less than $R$. In this case for small pitch angles $\left(\psi_{P}<1 / \gamma_{\|}\right.$, where $\gamma_{\|}=\left(1-\beta_{\|}^{2}\right)^{-1 / 2} \gg 1$, ( $c \beta_{\|}$- the guiding center velocity along field lines) the synchrotron radiation is replaced by a sum of the curvature and undulator radiation (Sobolev 1999). The term "undulator" radiation is used for a case when the radiation is coming from many electron spiraling around magnetic field line.

If the radiation is from a segment $\left(R / \gamma_{\|}\right)$of the trajectory, which contains $N>1$ cyclotron periods, then at small pitch angles the emitted spectrum consists of both broadband curvature radiation at low frequencies and relatively narrowband undulator radiation at higher frequencies (Fig.1). 
The power spectrum of undulator radiation at the first harmonic may be written down in the form

$$
\begin{aligned}
\frac{d P_{\pi}^{u}}{d \omega} & =\frac{3}{4} W \frac{\left(N \sin \psi_{P}\right)^{2}}{\left|\omega_{B}\right|} \xi\left(1-2 \xi^{1 / 2}+2 \xi^{3 / 2}\right) \theta(1-\xi), \\
\frac{d P_{\sigma}^{u}}{d \omega} & =\frac{3}{4} W \frac{\left(N \sin \psi_{P}\right)^{2}}{\left|\omega_{B}\right|} \xi \theta(1-\xi),
\end{aligned}
$$

where $\theta(x)=\{1, x \geq 0 ; 0, x<0\}, \xi=\omega /\left(2\left|\omega_{B}\right| \gamma_{\|}^{2}\right), \omega_{B}=e B /(\gamma m c), N=$ $\left|\omega_{B}\right| R /\left(\gamma_{\|} c\right), W=(2 / 3)\left(e^{2} / c\right)(c / R)^{2} \gamma_{\|}^{4}$ - the total power of the curvature radiation.

The total power of the undulator radiation emitted in $\pi$ - and $\sigma$-polarization components are $P_{\pi}^{u}=0.41 W\left(N \gamma_{\|} \sin \psi_{P}\right)^{2}$ and $P_{\sigma}^{u}=0.75 W\left(N \gamma_{\|} \sin \psi_{P}\right)^{2}$; though for synchrotron radiation $P_{\pi}=(1 / 8) W$ and $P_{\sigma}=(7 / 8) W$.

Fig. 1 shows the spectrum distributions of the undulator-curvature radiation. The first peak corresponds to curvature radiation, the second and third peaks are associated with the first and second harmonics of undulator radiation, respectively. Thus, in pulsar magnetosphere relativistic particles may lose energy by two emitting mechanisms: curvature radiation with a high degree of linear polarization and undulator mechanism with a high degree of circular polarization.
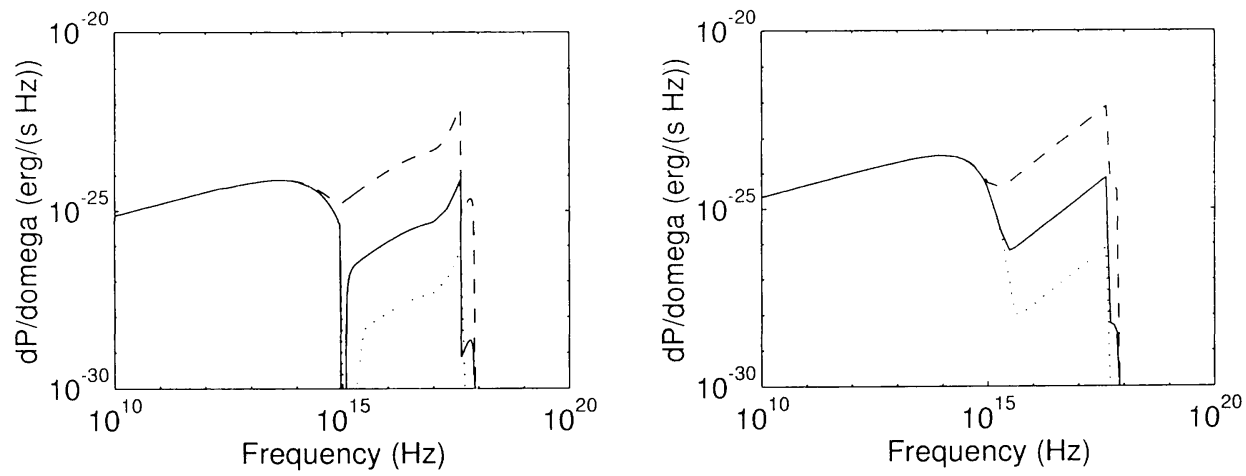

Figure 1. Spectrum distributions of undulator-curvature radiation for $\pi$ - (left) and $\sigma$ - polarizations (right); $B=10^{6} \mathrm{G}, R=10^{8} \mathrm{~cm}, \gamma_{\|}=$ $10^{4},\left(\right.$ and $\left.N=10^{3}\right)$; pitch angles: $\psi_{P}=0.1 / \gamma_{\|}\left(\right.$dashed curves); $\gamma_{\|} \psi_{P}=$ 0.01 (solid curves); $\gamma_{\|} \psi_{P}=0.001$ (dotted curves).

\section{References}

Cheng, K.S., \& Zhang, J.L. 1996, ApJ, 463, 271

Radhakrishnan, V. 1969, Proc. Astr. Soc. Austral., 1, 254

Radhakrishnan, V. \& Rankin, J.M. 1990, ApJ, 352, 258

Sobolev, Ya.M. 1999, Radio Phys. and Radio Astron., 4, 38 